\title{
From the Choice of a Regimen to the Choice of an Intensity: Changing Perspective in the Antithrombotic Therapy of Atrial Fibrillation Patients Undergoing Percutaneous Coronary Intervention
}

\author{
Andrea Rubboli ${ }^{1} \cdot$ Elena Barbaresi ${ }^{1} \cdot$ Bianca Rocca ${ }^{2}$ \\ Published online: 2 December 2019 \\ (C) Springer Science+Business Media, LLC, part of Springer Nature 2019
}

Over the past years, the issue of antithrombotic therapy in patients with atrial fibrillation (AF) undergoing percutaneous coronary intervention (PCI) has essentially focused on the contraposition between triple, i.e., oral anticoagulant (OAC), aspirin and clopidogrel, and double, i.e., OAC and clopidogrel, therapy as the treatment of choice [1,2]. Whereas uncertainty persists over whether the two regimes are comparably effective, there is no doubt that with triple therapy, the risk of major and/or total bleeding complications is higher than with double therapy $[1,2]$. In other words, a higher intensity of antithrombotic therapy, as a result of three antithrombotic agents given in conjunction, is less safe than a lower intensity, as obtained with the combination of only two antithrombotic drugs. Such correspondence appears evident when only warfarin as the OAC and clopidogrel as the $\mathrm{P} 2 \mathrm{Y}_{12}$-inhibitor are considered. This may not be the case when OAC safer than warfarin, including the non-vitamin $\mathrm{K}$-antagonist oral anticoagulants (NOAC) dabigatran, apixaban, edoxaban, and rivaroxaban, and $\mathrm{P} 2 \mathrm{Y}_{12}$-inhibitors more potent than clopidogrel, including prasugrel and ticagrelor, are given in combination. The intensity, and associated risk of bleeding, of double therapy with less safe agents, such as warfarin and prasugrel or ticagrelor, might actually be higher than that of triple therapy with safer drugs, such as NOAC, aspirin, and clopidogrel. Indeed, in a recent network meta-analysis, where 10,026 patients from WOEST [3], PIONEER AF-PCI [4], RE-DUAL PCI [5], and

Andrea Rubboli

andrea.rubboli@auslromagna.it

1 Department of Cardiovascular Diseases - AUSL Romagna, Division of Cardiology, Ospedale S. Maria delle Croci, Viale Randi 5,

48121 Ravenna, Italy

2 Institute of Pharmacology, Catholic University School of Medicine, Rome, Italy
AUGUSTUS [6] trials were included, the risk of major bleeding was shown to be progressively lower with triple therapy of NOAC, aspirin, and clopidogrel; double therapy of warfarin and clopidogrel; and double therapy of NOAC and clopidogrel, respectively, as compared with conventional triple therapy of warfarin, aspirin, and clopidogrel [7]. This means that reducing the overall intensity of antithrombotic therapy, by either keeping the regimen, i.e., triple, but using a safer OAC, i.e., NOAC instead of warfarin, or keeping the $\mathrm{OAC}$, i.e., warfarin, but de-escalating the regimen, i.e., double instead of triple, or changing both the regimen, i.e., double instead of triple, and the OAC, i.e., NOAC instead of warfarin, an increase in safety is obtained. The importance of the overall treatment intensity rather than the regimen per se is further supported by the results of the PIONEER AF-PCI trial [4], where with the same triple therapy regimen of OAC, aspirin, and clopidogrel, the use of very low-dose rivaroxaban $2.5 \mathrm{mg}$ twice daily instead of warfarin was associated with significantly less bleeding events.

There is currently little data on the individual contribution to the risk of bleeding of the more potent $\mathrm{P}_{2} \mathrm{Y}_{12}$-inhibitors prasugrel and ticagrelor when used in combined antithrombotic therapies. An increase in hemorrhagic complications has been reported in comparison with clopidogrel with the same antithrombotic regimen [8], but the safety impact of prasugrel or ticagrelor used for example in double therapy as compared with clopidogrel in triple therapy remains undetermined.

While acknowledging current uncertainties and waiting for further evidence, the different antithrombotic therapies may hypothetically be ranked as outlined in Fig. 1, based on their overall intensity (and associated risk of bleeding). For the time being, a perspective change from just the number of drugs to be used in combination to a more comprehensive evaluation of the overall treatment intensity is advised when aiming at optimal safety of antithrombotic therapy in AF patients undergoing PCI. 
Fig. 1 Hypothetical ranking of the different antithrombotic therapies for $\mathrm{AF}$ patients undergoing PCI, based on the overall intensity of treatment (and associated risk of bleeding). $\mathrm{AF}$ atrial fibrillation, PCI percutaneous coronary intervention, ASA aspirin, NOAC non-vitamin K-antagonist oral anticoagulant

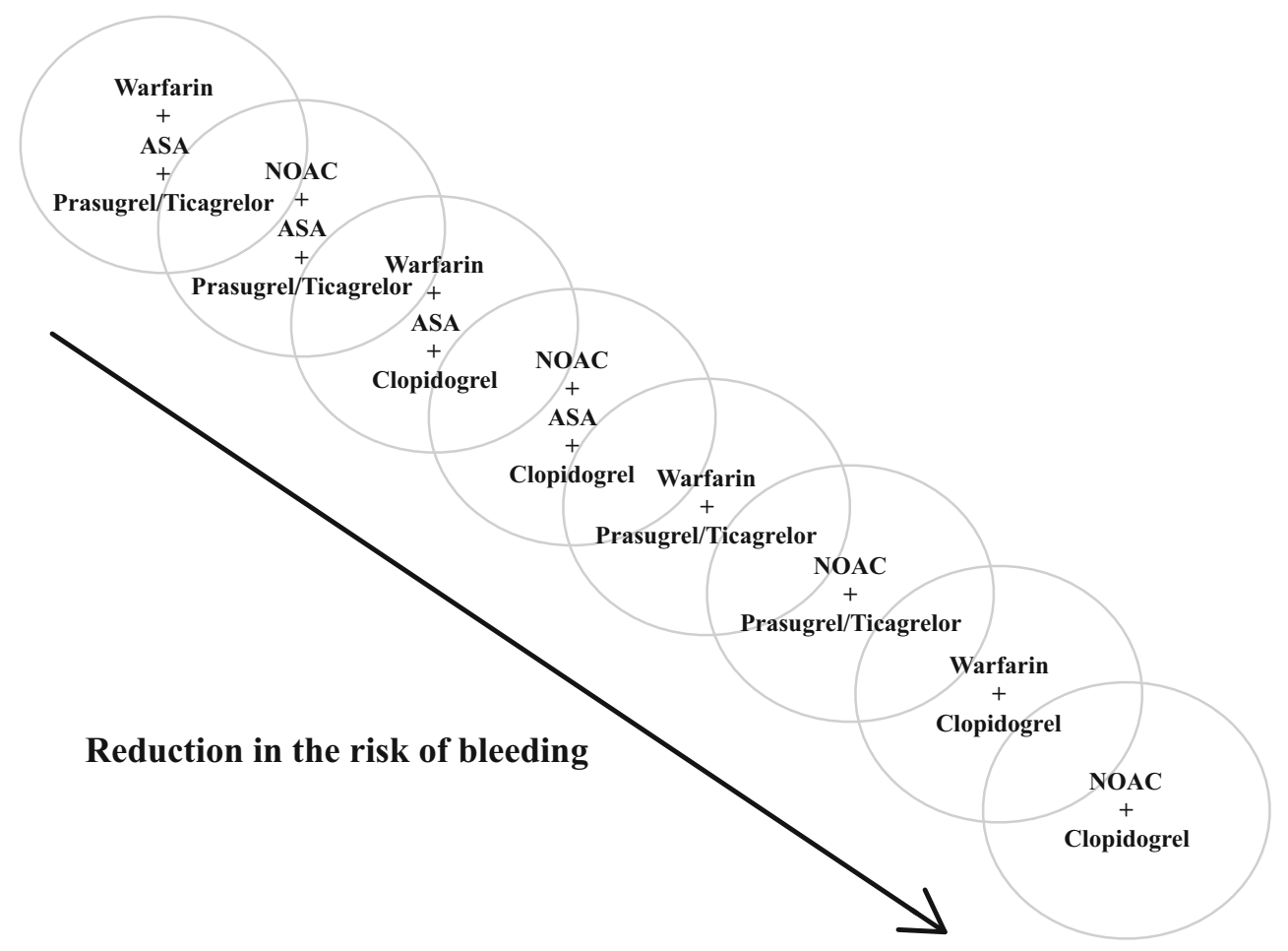

\section{References}

1. Lip GYH, Collet JP, Haude M, et al. 2018 Joint European consensus document on the management of antithrombotic therapy in atrial fibrillation patients presenting with acute coronary syndrome and/ or undergoing percutaneous cardiovascular interventions: a joint consensus document of the European Heart Rhythm Association (EHRA), European Society of Cardiology Working Group on Thrombosis, European Association of Percutaneous Cardiovascular Interventions (EAPCI), and European Association of Acute Cardiac Care (ACCA) endorsed by the Heart Rhythm Society (HRS), AsiaPacific Heart Rhythm Society (APHRS), Latin America Heart Rhythm Society (LAHRS), and Cardiac Arrhythmia Society of Southern Africa (CASSA). Europace. 2019;21:192-3.

2. Angiolillo DJ, Goodman SG, Bhatt DL, J, et al. Antithrombotic therapy in patients with atrial fibrillation treated with oral anticoagulation undergoing percutaneous coronary intervention. Circulation. 2018;138:527-36.

3. Dewilde WJ, Oirbans T, Verheugt FW, et al. WOEST study investigators. Use of clopidogrel with or without aspirin in patients taking oral anticoagulant therapy and undergoing percutaneous coronary intervention: an open-label, randomised, controlled trial. Lancet. 2013;381:1107-15.
4. Gibson CM, Mehran R, Bode C, W, et al. Prevention of bleeding in patients with atrial fibrillation undergoing PCI. N Engl J Med. 2016;375:2423-34.

5. Cannon CP, Bhatt DL, Oldgren J, T, et al. Dual antithrombotic therapy with dabigatran after PCI in atrial fibrillation. N Engl J Med. 2017;377:1513-24.

6. Lopes RD, Heizer G, Aronson R, et al. Antithrombotic therapy after acute coronary syndrome or PCI in atrial fibrillation. N Engl J Med. 2019;380:1509-24.

7. Lopes RD, Hong H, Harskamp RE, P, et al. Safety and efficacy of antithrombotic strategies in patients with atrial fibrillation undergoing percutaneous coronary intervention: a network meta-analysis of randomized controlled trials. JAMA Cardiol. 2019. https://doi.org/ 10.1001/jamacardio.2019.1880.

8. Jackson LR 2nd, Ju C, Zettler M, et al. Outcomes of patients with acute myocardial infarction undergoing percutaneous coronary intervention receiving an oral anticoagulant and dual antiplatelet therapy: a comparison of clopidogrel versus prasugrel from the TRANSLATE-ACS study. JACC Cardiovasc Interv. 2015;8:1880-9.

Publisher's Note Springer Nature remains neutral with regard to jurisdictional claims in published maps and institutional affiliations. 